\title{
Simulation for Prediction of Entry Article Demise (SPEAD): an Analysis Tool for Spacecraft Safety Analysis and Ascent/Reentry Risk Assessment
}

Author: Dr. Lisa Ling, Lisa.M.Ling@nasa.gov

NASA Johnson Space Center, 2101 NASA Road One, Houston, TX 77058

For the purpose of performing safety analysis and risk assessment for a probable offnominal suborbital/orbital atmospheric reentry resulting in vehicle breakup, a synthesis of trajectory propagation coupled with thermal analysis and the evaluation of node failure is required to predict the sequence of events, the timeline, and the progressive demise of spacecraft components. To provide this capability, the Simulation for Prediction of Entry Article Demise (SPEAD) analysis tool was developed. This report discusses the capabilities, modeling, and validation of the SPEAD analysis tool.

SPEAD is applicable for Earth or Mars, with the option for 3 or 6 degrees-of-freedom (DOF) trajectory propagation. The atmosphere and aerodynamics data are supplied in tables, for linear interpolation of up to 4 independent variables. The gravitation model can include up to 20 zonal harmonic coefficients. The modeling of a single motor is available and can be adapted to multiple motors. For thermal analysis, the aerodynamic radiative and free-molecular/continuum convective heating, black-body radiative cooling, conductive heat transfer between adjacent nodes, and node ablation are modeled. In a 6DOF simulation, the local convective heating on a node is a function of Mach, angle-ofattack, and sideslip angle, and is dependent on 1) the location of the node in the spacecraft and its orientation to the flow modeled by an exposure factor, and 2) the geometries of the spacecraft and the node modeled by a heating factor and convective area. Node failure is evaluated using criteria based on melting temperature, reference heat load, g-load, or a combination of the above. The failure of a liquid propellant tank is evaluated based on burnout flux from nucleate boiling or excess internal pressure. Following a component failure, updates are made as needed to the spacecraft mass and aerodynamic properties, nodal exposure and heating factors, and nodal convective and conductive areas. This allows the trajectory to be propagated seamlessly in a single run, inclusive of the trajectories of components that have separated from the spacecraft. The node ablation simulates the decreasing mass and convective/reference areas, and variable heating factor. A built-in database provides the thermo-mechanical properties of common spacecraft materials and propellants. Monte Carlo analysis can be performed and the debris footprint plotted on a world map. A scheduler is available to set up userdefined events. The software architecture consists of modularized functions designed to allow quick incorporation of guidance, navigation, control, and mechanical models.

The software and methodology have been validated against actual flights, telemetry data, validated software, independent analyses by other organizations, and manual calculations. The application of SPEAD includes the analysis of Stardust's probable off-nominal Earth-return reentry; reconstruction of Soyuz $14 \mathrm{~S}$ and $15 \mathrm{~S}$ ballistic reentries; OsirisREx's probable off-nominal Earth-return reentry; suborbital reentry of Falcon 9 Upper Stage with Dragon capsule from potential launch failure; and Boeing CST-100 service module’s nominal reentry breakup analysis. 